\title{
PREVENCIÓN, LA MEJOR ARMA PARA GANAR LA GUERRA AL FRAUDE
}

Prevention, the best weapon to win the war on fraud

\author{
Maricella Corpas Ford \\ Facultad de Administración de Empresas y Contabilidad, Universidad de Panamá \\ Email: maricellarosario01@hotmail.com iD https://orcid.org/0000-0001-6430-4423
}

\section{RESUMEN}

La presente investigación de enfoque descriptivo explicativo con carácter documental, pretende un análisis critico de a través de un tema que data de un tiempo pero que sigue siendo actual: EL FRAUDE, desde la visión de la prevención como mejor de arma para ganar la guerra a este flagelo, que afecta la dinámica financiera, la transparencia, la imagen, la ética y la moral de instituciones grandes y pequeñas.

Se presenta como una reflexión que supone conseguir armas o anillos de seguridad contra el fraude, desde el análisis como un todo, de una manera global e integrada, ya que desde este punto de vista la prevención es de todos y un trabajo diario que no debe descansar, aplicado la prevención, en todas las áreas y procedimientos, y avizorar, anteponerse a los hechos desviados.

Los anillos de seguridad como así se han definido en este análisis, son justos y necesarios pues el mundo actual, más competitivo, tecnológico y globalizad nos coloca frente a grandes desafíos, como el flagelo del fraude; su impresionante evolución, perfeccionamiento y alcance, son aspectos medulares que requieren de esfuerzo, planificación, análisis y concienciación para poder superarlo. Es así como, finalmente luego del análisis bibliográfico documental y estadísticos que existe en la web, aunado a la propia realizada nos arroja eque la mejor herramienta contra el fraude es la prevención.

Palabras claves: Anillos, seguridad, flagelo, avizorar.

\section{ABSTRACT}

The present research of a descriptive explanatory approach with a documentary character, aims at a critical analysis of a topic that dates back to a time but that is still current: FRAUD, from the vision of prevention as the best weapon to win the war against this scourge, which affects the financial dynamics, transparency, image, ethics and morale of large and small institutions.

It is presented as a reflection that involves obtaining weapons or security rings against fraud, from the analysis as a whole, in a global and integrated way, since from this point of view prevention is everyone's and a daily work that should not rest, applied prevention, in all areas and procedures, and watch, take precedence over deviant events.

Security rings, as defined in this analysis, are fair and necessary since today's more competitive, technological and globalized world places us in front of great challenges, such as the scourge of fraud; Its impressive evolution, improvement and scope are core aspects that require effort, planning, analysis and awareness to overcome it. This is how, finally after the documentary and statistical bibliographic analysis that exists on the web, together with the one carried out, it shows us that the best tool against fraud is prevention.

Keywords: Rings, security, scourge, watch. 
REVISTA FAECO SAPIENS

ISSN L 2644-3821

Acceso Abierto. Disponible en: https://revistas.up.ac.pa/index.php/faeco_sapiens

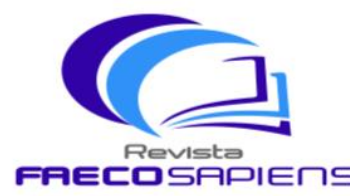

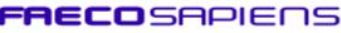

\section{INTRODUCCIÓN}

El mundo actual nos coloca frente a grandes desafíos, como el flagelo del fraude; su impresionante evolución, perfeccionamiento y alcance, son aspectos medulares que requieren de esfuerzo, planificación, análisis y concienciación para poder superarlo.

El fraude, desde un contexto amplio abarca el engaño para configurarlo como delito, a través del cual se obtiene una ganancia, pues, el fraude tal como lo indica Black's Law Dictionary es: "Una declaración falsa a sabiendas de la verdad o la ocultación de un hecho material para inducir a otro a actuar a en su detrimento", partiendo de esta definición concreta y entendible par este complejo delito, al que no se escapan instituciones, empresas, organizaciones, la sociedad en pleno; surge la preocupación para evitarla.

Los fraudes afectan en gran medida los procesos administrativos de cualquier actividad, una vez que se establece, siendo muy variados los motivos que le originan y permite y pocos los involucrados pero muchos los afectados; donde sorprende a veces las causas que lo motivan, tal como lo declara (Orozco, 2019) que una de las motivaciones para cometer la falta, no es la respuesta clásica como "por problemas económicos", sino: "porque pude hacerlo" o "la ventana estaba abierta", es decir la oportunidad.

De allí, la importancia y preocupación en la búsqueda de herramientas que le minimicen, en conseguir "una fórmula" que vaya más allá del control, del seguimiento, es pensar que es posible que se erradique y se evite su institucionalización, si se le hace un frente, que desde esta perceptiva la mejor arma es la prevención desde un todo y para todos., es anticiparnos para que no sucedan los efectos negativos, es estar preparados, es tener el arma preparada atacar y ganar. 
REVISTA FAECO SAPIENS

ISSN L 2644-3821

Acceso Abierto. Disponible en https://revistas.up.ac.pa/index.php/faeco_sapiens

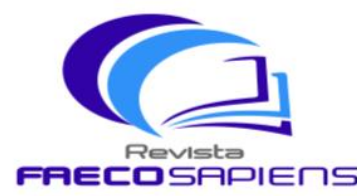

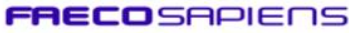

\section{MATERIALES Y MÉTODOS}

La presente investigación, se desarrolla en el contexto actual geográfico de Panamá, bajo un diseño bibliográfico y documental, en base a una metodología descriptiva con su consecuente análisis, para lo cual, lo haremos partiendo del concepto de Fraude que nos facilita el Diccionario de la Real Academia (RAE), según sus términos fraude es la: "acción contra la verdad y la rectitud que perjudica a la persona contra quien se comete" (RAE, 2020). De allí, se desprenden elementos que nos permite contrastar algunas causas que originan el mismos, como los valores éticos morales, pues enlaza la ausencia o poca verdad y rectitud claves para que se configure el fraude, así como la intención, ya que es una acción premeditada para perjudicar; por otro lado, causa un provecho, supone entonces engaño, es decir falsear, omitir datos o verdades.

Así mismo, según la ACFE (Association of Certified Fraud Examiners): ACFE (2014), el fraude es cualquier actividad o acción con el propósito de enriquecimiento personal a través del uso inapropiado de recursos o activos de una organización por parte de una persona.

De esta manera, es común oír hablar sobre fraude, en cualquier instancia, de su variedad alcance y perfeccionamiento; de los efectos negativos a la gestión, a la imagen, a la credibilidad de organizaciones, empresas, instituciones e incluso a la sociedad, esto conlleva a afirmar que prácticamente el fraude existe en todo. Ello amerita, establecer algunas estrategias o herramientas, sí deseamos ciertamente enfrentarlo y ganarle la guerra, para detectar vicios o señales que nos den la alerta, que nos avizoren, adviertan la posible comisión del delito de fraude.

Es menester amalgamar, conceptos, procedimientos, técnicas, pruebas, análisis proactivos que nos facilite la identificación de lo obvio, de los riesgos potenciales, de las irregularidades, pero todo ello desde la propia prevención, la evitación a la 
REVISTA FAECO SAPIENS

ISSN L 2644-3821

Acceso Abierto. Disponible en: https://revistas.up.ac.pa/index.php/faeco_sapiens

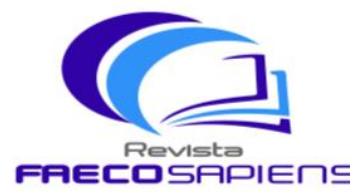

คคEСロらคคIEกร
Volumen 3 - Número 2 Julio-Diciembre 2020

Recibido: 11/05/20 Aceptado: 11/06/20 pp. 84-95 Indexada

ocurrencia, de manera que sabiendo que, se han establecido muchas herramientas para evitar el fraude, es hora, entonces de concretar, predecir y evitarlo.

Dentro de las herramientas para evitar el fraude, independientemente de su tipo, se pueden mencionar en líneas generales:

\section{Auditoria:}

Existen muchos tipos de auditoría, pero es la auditoría interna, la que es utilizada para determinar el cumplimiento de los objetivos, evaluar o mejorar procesos de gestión de riesgos y control a fin de hacerlos más eficaces dentro de cualquier dependencia llámese, organización, empresa o institución, en este sentido la auditoría interna ha sido definida por innumerables estudiosos e instituciones relacionadas con el tema, es así como el Instituto Norteamericano de Contadores Públicos (AICPA, 2008), menciona es: "Un examen que pretende servir de base para expresar una opinión sobre la razonabilidad, consistencia y apego a los principios de contabilidad generalmente aceptados, de estados financieros preparados por una empresa o por otra entidad para su presentación al Publico o a otras partes interesadas"

A ello se agrega que, si se trata de una expresión de racionalidad, esto debe implicar la advertencia, avizorar cualquier indicio que infiera inconsistencia, donde esa inconsistencia puede significar desviaciones, omisiones, falsedades; de allí deviene la importancia y la objetividad en el proceso de auditoría.

En otras palabras, la auditoria sería la primera herramienta o el "primer anillo de seguridad" como actividad independiente y objetiva que prevenga la comisión de fraudes, ante lo que es preciso mencionar que, esto es independiente del tipo de auditoría que se realice, pues su esencia y objetivo en concreto es el mismo, determinar la veracidad de los datos que se audita. 
REVISTA FAECO SAPIENS

ISSN L 2644-3821

Acceso Abierto. Disponible en: https://revistas.up.ac.pa/index.php/faeco_sapiens

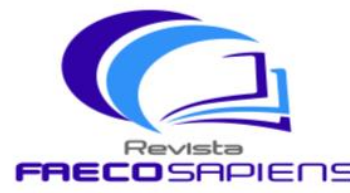

ศคEСロらคคIEாร

El Control Interno, así entonces hablemos del control interno, a sabiendas que en los últimos tiempos toda pequeña o gran organización se han interesado en su implementación, como herramienta de primera mano para prevenir el fraude, en sus procesos, bien sea por propios o externos. Pero que significa este según (Araujo, 2008) "Los controles internos corresponden a los planes de organización y al conjunto coordinado de métodos y medidas adoptadas para salvaguardar sus activos, verificar la exactitud y la fiabilidad de los datos contables promover la eficiencia operativa y fomentar la adhesión a las directrices establecidas por la dirección de la empresa."

Se comprende que un bien control interno, pone énfasis en la evaluación que conlleva a todo un proceso que comienza con una comprensión, continúa con una evaluación preliminar, pruebas de cumplimiento, revaluación de los controles,

Sin tecnicismos, es la vigilancia interna, en íntimo de la empresa, es tener encendidas las 24 horas el radar de la propia institución, donde los principales responsables debe ser la alta gerencia, pero involucrada toda la organización, es decir es un trabajo de todos en pro de la transparencia, efectividad, que conlleva al éxito de la gestión, que su éxito reflejará el verdadero trabajo en equipo.

Desde esta visión, no se pretende ser exagerado al contextualizar el fraude y la guerra que debemos librar en contra de él, pues este nos enfrenta a grandes desafíos, nuevas formas, trasmuta, se prolifera, se reinventa, todo ello gracias a la evolución tecnología, la apertura de nuevas formas de negocios, que confluye en la globalización.

Por otra parte, es necesario mencionar que según, Church (1996) existen diversos estudios que se centran en determinar cómo el auditor interno puede establecer que existe alguna irregularidad en la información financiera llevando a cabo pruebas analíticas, lo que evidencia, que los auditores internos son sensibles a factores que 
REVISTA FAECO SAPIENS

ISSN L 2644-3821

Acceso Abierto. Disponible en

https://revistas.up.ac.pa/index.php/faeco_sapiens

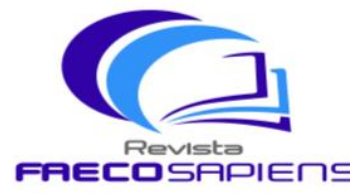

คคEСロらคคIEกร
VIV Volumen 3 - Número 2 Julio-Diciembre 2020

Recibido: 11/05/20 Aceptado: 11/06/20 pp. 84-95 Indexada

afectan la posibilidad de fraudes en la información financiera, quiere decir que están atentos, alertas y realizan lo pertinente para detectar el fraude

Entonces, in un verdadero redimensionamiento, actualización, sin bases sólidas, y una mejor visión que implique no solo procesos internos, si no estar vigilantes a todo el entorno o ambiente de los que forman parte de la organización, incluida la comunicación interna, si se espera que el control interno que nos provea de información esencialmente importante en donde se fundamenten las decisiones importantes, ¿para que el control interno? Esa es la esencia.

Este debe ser sincero, libre de las omisiones, correcciones, ajustes no acordes, es decir libre de fraudes contables que afecten dichos resultados y por ende la información, entonces el control interno como proceso propio representa el "segundo anillo de seguridad ", para enfrentar los fraudes, ya que este permite una rápida detección y corrección de fallos y desajustes en sus diferentes procesos, pero dependerá en gran parte de su buena segmentación, separación de funciones, asignación de responsabilidades, y el necesario seguimiento continuo, que es un fundamento de la prevención.

\section{Ética Vs Nivel socioeconómico}

El fraude comporta, una conducta contraria a normas y preceptos éticos, en cualquier ámbito representa una grave amenaza para el avance y desarrollo de países e instituciones tanto privadas como públicas, que están preocupadas en combatir este tipo de conducta. De manera que, la prevención desde un enfoque moral y ético, debe orientarse en sensibilizar, en resaltar lo ético, y no sucumbir a las tentaciones de dadivas o privilegios $u$ otros artificios que seduzcan; desde la perspectiva humana tenemos la capacidad de evitar generar oportunidades adversas que conlleve a que se cometa el fraude, consiste en evitar que genere la oportunidad. 
REVISTA FAECO SAPIENS

ISSN L 2644-3821

Acceso Abierto. Disponible en: https://revistas.up.ac.pa/index.php/faeco_sapiens

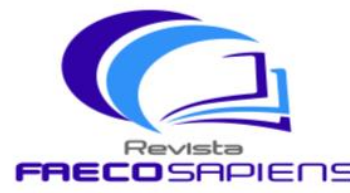

ศคEСロらคคIEாร

Esta oportunidad, muchas veces es movida por el componente socioeconómico que influye en los comportamientos y necesidades personales, es allí, donde se debe poner especial atención, y promover constantemente, motivar y retribuir al personal.

Ciertamente, el fraude es un hecho pernicioso que afecta varias esferas: lo económico, lo político, lo social, lo cultural, lo humano; y desde esa perspectiva de lo humano se trastoca lo ético y al amoral de las personas que se involucran a través de varias acciones como el soborno que los corrompe, convirtiéndose en algo que es catalogado más allá de las normas legales en un acto ilícito que puede ser un delito, que no escapa del juicio de valor que otorga la sociedad como algo inmoral.

Este juicio social, que va más allá de lo jurídico, te condena sin ser extremistas hasta el final, pues al no establecer una pena como tal, se te encasilla y está siempre latente, se pierde credibilidad, es un juicio inquisidor, condenatorio, y convierte al individuo éticamente reprobable.

Dentro de este marco, (Rabazo, 2017) establece que debe existir un comportamiento y compromiso ético de toda la institución, como pilar sobre el que se asiente cualquier programa anti-fraude, ya que la atmosfera que se perciba, debe ser clara sin duda respecto al comportamiento ético; para que cualquier esfuerzo, que se efectúe para establecer controles, sea efectivo.

En efecto sobre la base de cualquier código, política, guía, manual o procedimiento deben estar sustentados principios éticos, que se vincula fraude con el comportamiento contrario a lo ético. Vista la ética como valor y forma para controlar esa conducta intencional, resaltando entonces que el fraude es éticamente inaceptable.

Pero entonces, ¿qué significa la moral ante el fraude?, es el juicio de valor que de alguna manera servirá de medida o freno para esa conducta impropia y reprochable, que guía al hombre a su conducción ante la vida con dignidad donde el valor moral 
REVISTA FAECO SAPIENS

ISSN L 2644-3821

Acceso Abierto. Disponible en: https://revistas.up.ac.pa/index.php/faeco_sapiens

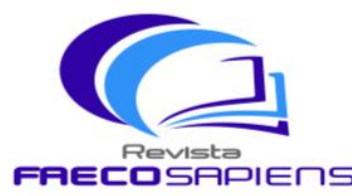

คคEСロらคคIEกร

arrastra al bien moral, lo perfecciona en voluntad y razón. En este sentido una moral solida contribuye en la lucha contra este flagelo del fraude entre particulares y las administraciones del Estado.

A sabiendas que, existen varios valores morales que conforman los valores de una persona nos referiremos solo a: respeto, dignidad y honestidad; básicos en todo nuestro actuar como personas, pero indispensable e ineludibles en el entorno laboral como parte de un engranaje para conformar conductas probas.

Cabe destacar que, resaltando lo ético en armonía con los componentes socioeconómicos atendidos cuidadosamente, desde esta perspectiva, se forma el tercer anillo de seguridad de prevención para evitar el fraude.

Imperioso entonces, resaltar valores como la idoneidad, conceptualizada esta como aptitud técnica, legal y moral, como condición primordial para acceder a la función pública, igualmente y en comparación con los valores propios de la moral, establece el principio de responsabilidad como obligación para cumplir intachablemente sus deberes, incluso establece esa responsabilidad directamente proporcional, pues mientras más elevado sea el cargo que ocupa, mayor deberá ser la responsabilidad asumida.

Como se ha observado, existen múltiples factores que provocan los frades, de manera que allí es donde radica la importancia de la prevención, como herramienta en contra del fraude, tal como lo mencionan en su estudio DeFond y Jiambalvo (1991), existen tres factores que provocan que los errores (fraudes), intencionados o no en la información financiera, y son los siguientes la falta de control, la falta de auditoria y el ambiente de control de la institución, pues en control su existencia ya la probabilidad reduce la probabilidad de errores.

Esta postura, convalida lo expuesto hasta aquí pues, combinan entonces estos factores y establecen que inciden en una mayor fiabilidad de la información 
REVISTA FAECO SAPIENS

ISSN L 2644-3821

Acceso Abierto. Disponible en https://revistas.up.ac.pa/index.php/faeco_sapiens

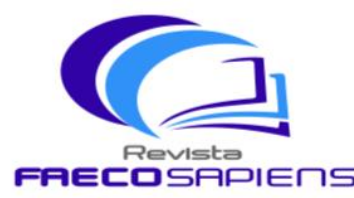

คคEСロらคคIEกร

financiera y esto tiene su reflejo en la prevención al fraude, ya que al facilitar información fiable se aleja de cualquier indicio de fraude.

Todo ello nos hace repensar todo sobre los medios para prevenir el fraude, existen métodos, formas, pero insistimos que lo fundamental es la Prevención, e insistimos en la guerra frontal contra este flagelo, resaltando principios de Sun Tzu quien escribió hace más de dos mil años los textos que dan vida al libro El Arte de la Guerra, las connotaciones remitían a estrategias militares, pues bien, los actuales momentos sabemos que nos indican que las luchas frontales, son guerras como el caso contra del fraude, una de las notas que resalan en esta lectura que se trae a colación para engrana parte de lo expresado es: "La doctrina: Organizar, Autoridad, promoción, vigilancia y necesidades." (Tsu, 2003) p. 5.

De lo que se desprende que, vigilar es ineludible, para hacer frente al que se decide enfrentarse, desde esa perspectiva de estrategias, es cerrar las oportunidades, las causas y la mejor es: prevenir.

Entonces, prevenir en sentido amplio es tomar precauciones o medidas por adelantado para evitar un daño, un riesgo o un peligro, es adelantarse a un hecho generalmente aceptado como negativo o perjudicial, pernicioso, para que no ocurra.

Según el u origen es el término del latín praeventǐo, el cual proviene de "prae": previo, anterior, y "eventious": evento o suceso. (Definiciones.com)

En tanto, al vincular con hechos de fraudes, será entonces la estrategia para vigilar, adelantarnos, evitar un riesgo, daño, prever que ocurra, donde esta acción es de todos. 
REVISTA FAECO SAPIENS

ISSN L 2644-3821

Acceso Abierto. Disponible en

https://revistas.up.ac.pa/index.php/faeco_sapiens

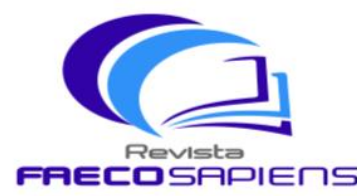

Recibido: 11/05/20 Aceptado: 11/06/20 pp. 84-95 Indexada

\section{RESULTADOS Y DISCUSIÓN}

A continuación, se presenta una breve entrevista, que pretende determinar si a través de la adopción de normas, políticas y procedimientos, se pueda prevenir la ocurrencia de fraudes: A través de la opinión de los participantes, se determina que el $100 \%$ considera que el tema es actual y relevante. Se puede evidenciar que, el $56 \%$ de los participantes considera que, si existen mecanismos legales suficientes para evitar el fraude, mientras que el $44 \%$ no lo cree.

\section{Herramientas paera prevenir el Fraude}

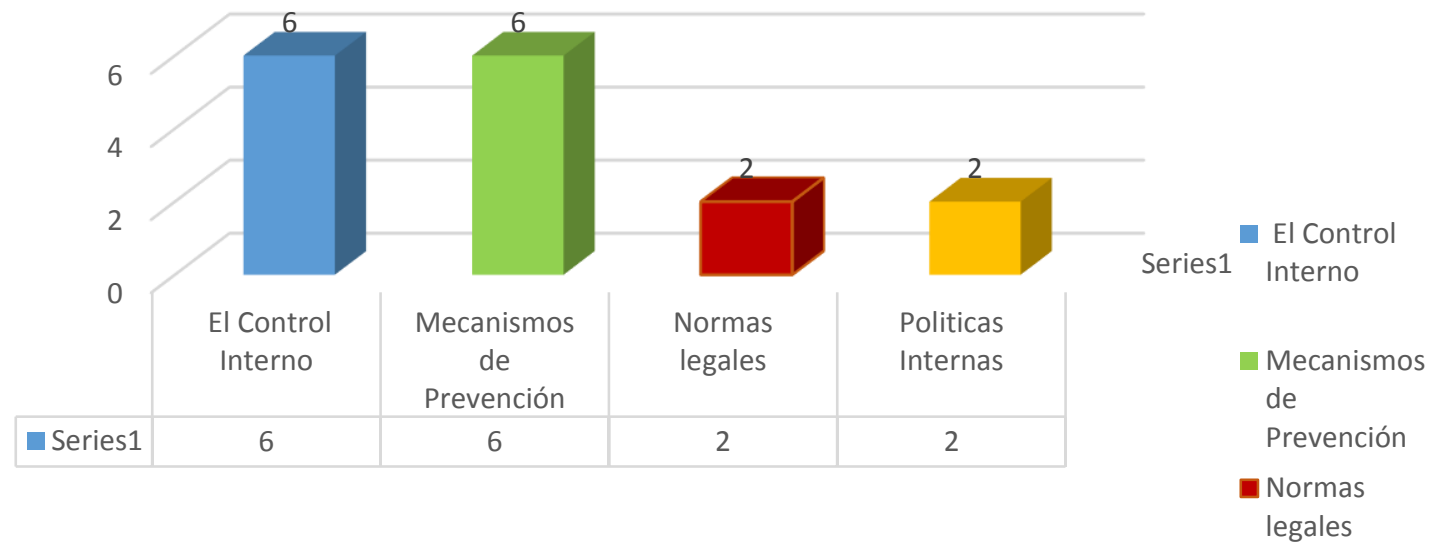

Gráfico 1: Herramientas para evitar el Fraude

Fuente: Encuestados por la autora

El 87\%, declara que ha escuchado sobre fraudes en su entorno, entendiendo que puede ser en sus áreas de trabajo, comunidad, o grupos afines. En este caso el 75 $\%$ dio la máxima puntuación, afirmando que, si se puede prevenir el fraude, por otro lado, el $25 \%$ cree que en algunas ves si se puede.

En cuanto esta consideración control interno y los mecanismos de prevención lograron obtener un puntaje igual, es decir un 37\% por ambos lados, así mismos 
REVISTA FAECO SAPIENS

ISSN L 2644-3821

Acceso Abierto. Disponible en:

https://revistas.up.ac.pa/index.php/faeco_sapiens

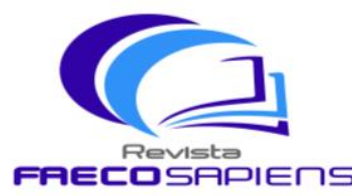

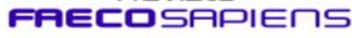

Volumen 3 - Número 2 Julio-Diciembre 2020

Recibido: 11/05/20 Aceptado: 11/06/20 pp. 84-95 Indexada

normas legales y políticas internas $13 \%$ cada una, lo que quiere decir, que los encuestados consideran en líneas generales que si hay herramientas y si se pude evitar el fraude.

\section{CONCLUSIONES}

Necesario e imperioso, resulta entonces el reordenamiento de prácticas actuales, afianzar y redimensionar la supervisión interna, mantenerse en vigilia, rigurosa. Sin embargo, sostenemos que, en adición, debe existir un cambio de actitud frente al fraude, para poder reducir la recurrencia. Ponderar la ética y considerar los aspectos socioeconómicos, valorar y recocer, denunciar e identidad del acto de fraude.

Definitivamente siempre será la prevención la mejor arma para enfrentar la guerra al delito del fraude. Pues la prevención nos antepone a la ocurrencia, es tomar las medidas necesarias para evitar que se materialice detectar la oportunidad de fraude y la anula.

Es mejor prevenir para contrarrestar o mitigue los hechos contrarios, en todo caso, urge combinar medidas de prevención, disuasión y detección, resaltando la conveniencia de las acciones preventivas. Finalmente se debe combinar, auditoria, control interno, ética y valores, adicionalmente establecer acciones para la prevención, detección y mitigación de actos ilícito en que se conforma el fraude.

\section{REFERENCIAS BIBLIOGRÁFICAS}

Abuse"., A. ". (2014). "Report to the Nations on occupational fraud and Abuse". Recuperado el 28 de 02 de 2020, de "Report to the Nations on occupational fraud and Abuse".: https://accid.org/wpcontent/uploads/2018/11/Senales_de_alerta_para_la_deteccion_de_fraude_en_las_empresa s.pdf

AICPA, I. F. (2008). Normas Internacionales de Auditoria. Recuperado el 28 de 02 de 2020, de Normas Internacionales de Auditoria: http://www.ifac.org/ 
REVISTA FAECO SAPIENS

ISSN L 2644-3821

Acceso Abierto. Disponible en:

https://revistas.up.ac.pa/index.php/faeco_sapiens

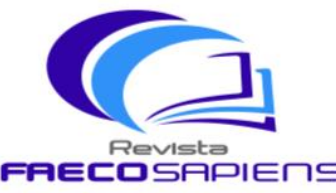

तार Volumen 3 - Número 2 Julio-Diciembre 2020

ismeli.co

Recibido: 11/05/20 Aceptado: 11/06/20 pp. 84-95

Indexada

Araujo, F. D. (2008). "La estructura de los controles internos y la independencia de la auditoría interna": Recuperado el 28 de 02 de 2020, de "La estructura de los controles internos y la independencia de la auditoría interna":: http://fccea.unicauca.edu.co/old/tgarf/tgarfse85.html

$\mathrm{CHURCH}$, B. K. (1995). 'Internal auditors' memory for financial-statement. Behavioral Research in Accounting, Vol. 7, pp. 17-36. Recuperado el 28 de 02 de 2020, de "Internal auditors' memory for financial-statement. Behavioral Research in Accounting, Vol. 7, pp. 17-36.: http://www.aeca1.org/pub/on_line/comunicaciones_aal2011/cd/30a.pdf

Definiciones.com. (s.f.). Concepto de Prevención. Recuperado el 28 de 02 de 2020, de Concepto de Prevención: Fuente: https://concepto.de/prevencion/\#ixzz6Ff4e5il8

DEFOND, M. y. (1991). "Incidence and Circumstances of Accounting Errors",. Recuperado el 28 de 02 de 2020, de "Incidence and Circumstances of Accounting Errors",: https://www.jstor.org/stable/247814

Orozco, F. (17 de 06 de 2019). ¿Por qué ocurren los fraudes? Recuperado el 28 de 02 de 2020, de ¿Por qué ocurren los fraudes?: https://www.elfinanciero.com.mx/monterrey/por-que-ocurrenlos-fraudes

Rabazo, A. (. (2017). El fraude Contable, una evidencia Empirica. Recuperado el 28 de 02 de 2020, de El fraude Contable, una evidencia Empirica: http://dehesa.unex.es/handle/10662/5587

RAE, D. d. (2020). Definción de la palabra Fraude. Recuperado el 28 de 02 de 2020, de Definción de la palabra Fraude: https://dej.rae.es/lema/fraude

Tsu, S. (2003). El arte de la Guerra. Recuperado el 02 de 28 de 2020, de El arte de la Guerra: https://www.biblioteca.org.ar/libros/656228.pdf 